\title{
Electron Microscopy Characterization of the Synergistic Effects between Pd, Au NPs, and Their Graphene Support
}

\author{
Kavita Meduri ${ }^{1}$, Candice Stauffer ${ }^{2}$, Graham O'Brien Johnson ${ }^{3}$, Paul G. Tratnyek ${ }^{3}$ and Jun Jiao ${ }^{1,2}$ \\ 1. Department of Mechanical \& Materials Engineering, Portland State University, Portland, USA. \\ 2. Department of Physics, Portland State University, Portland, USA. \\ 3. Institute of Environmental Health, Oregon Health \& Science University, Portland, USA.
}

There has been a growing interest in the synthesis of palladium (Pd) and gold ( $\mathrm{Au}$ ) nanoparticles (NPs) with graphene support as a catalyst for several important reactions [1], specifically for water treatment [2]. While various studies documenting the effects of Pd and Au using spectroscopy techniques such as UV-Vis, XPS, XRD etc. [1]-[3], and computational simulations [4], a systematic investigation of the synergistic effects of these metals using electron microscopy is missing. Reports suggest that during sonication, $\mathrm{Pd}$ and $\mathrm{Au}$ do interact with each other [1], [3], yet little has been confirmed about this interaction. To better understand the effect between these metal NPs and their support, an initial investigation of the interaction between $\mathrm{Pd}, \mathrm{Au} \mathrm{NPs}$, and graphene was carried out and reported here. The samples were synthesized by a combination of sonication and solvothermal processes [3], [5], [6]. The samples were characterized by an FEI Tecnai F-20 TEM/STEM with EDS capabilities.

Note that in the image of the sample made using both Pd and Au on graphene in Fig 1 (a), a cluster of smaller 2-5 nm Pd NPs appears to surround an Au core. The Au core is formed by a group of several smaller 20-100 nm Au NPs. In Fig 1 (b), these small Pd NPs are dispersed evenly over the graphene support, but no large clusters are formed in the absence of Au. Interestingly, for Fig 1 (c), the weight of the Au precursor was nearly doubled, and the amount of acetone solvent reduced by nearly ten times as much as that used in Fig 1 (a). Despite this, very few, small Au NPs in the range of 5-10 nm were formed on the graphene. When the original concentration was used, although a colour change was detected in the forming solution (indicating a reaction had occurred), no NPs were observed on the graphene. This is good agreement with a previous report that used DFT simulations [4], in which Au was shown to have a much weaker interaction with graphene than that of Pd. Our results are also in line the observation that formation of the $\mathrm{Pd} / \mathrm{Au}$ cluster is due to attractive interactions between $\mathrm{Pd}$ and $\mathrm{Au}$ atoms, which causes them to spontaneously move towards each other [4]. In addition, these images demonstrate that Pd has a great affinity for graphene, which correspondingly explains why the $\mathrm{Pd} / \mathrm{Au} \mathrm{NP}$ clusters cling to graphene. The $\mathrm{Pd}_{\text {shell }} \mathrm{Au}_{\text {core }}$ structure was found to have high surface energy stability over the $\mathrm{Au}_{\text {shell }} \mathrm{Pd}_{\text {core }}$ structure [1]. Conforming to this, no $\mathrm{Au}_{\text {shell }} \mathrm{Pd}_{\text {core }}$ structures were observed in these experiments. In Fig 2 (a), taken from an edge on the Pd/Au cluster in inset Fig 2 (b), the Pd NPs which group around the Au core were observed to have lattice structures and hence were determined to be crystalline in nature. Since Pd NPs cover the core, it is harder to analyse the Au NPs; however, at other locations on the sample (not shown here), lattice structures were observed on exposed Au cores. Similarly, the crystallinity of the NPs can be observed in Figs 2 (c) and (d). The dark-field STEM image can be seen in Fig 3 (a). The EDS maps of this image are shown in Fig 3 (b) for Pd and (c) for Au. Together, the maps establish that the cluster of smaller NPs are made up of Pd and surround a larger Au core.

In sum, these experiments and characterizations were able to demonstrate various structural phenomena that were speculated previously concerning the interactions between Pd, Au NPs, and graphene [7]. 


\section{References:}

[1] R Ferrando, J Jellinek, and RL Johnston, Chem. Rev. 108 (2008), p. 845.

[2] BP Chaplin et al, Environ. Sci. Technol. 46 (2012), p. 3655.

[3] Y Mizukoshi et al, J. Phys. Chem. B 101 (1997), p. 7033.

[4] W Ji et al, RSC Adv. 4 (2014), p. 55781.

[5] K Meduri et al, Microsc. Microanal. (2017), p. 1916.

[6] K Meduri et al, Microsc. Microanal. (2016), p. 332.

[7] The authors acknowledge funding from NSF award \#1507707, ONAMI and BUILD EXITO.
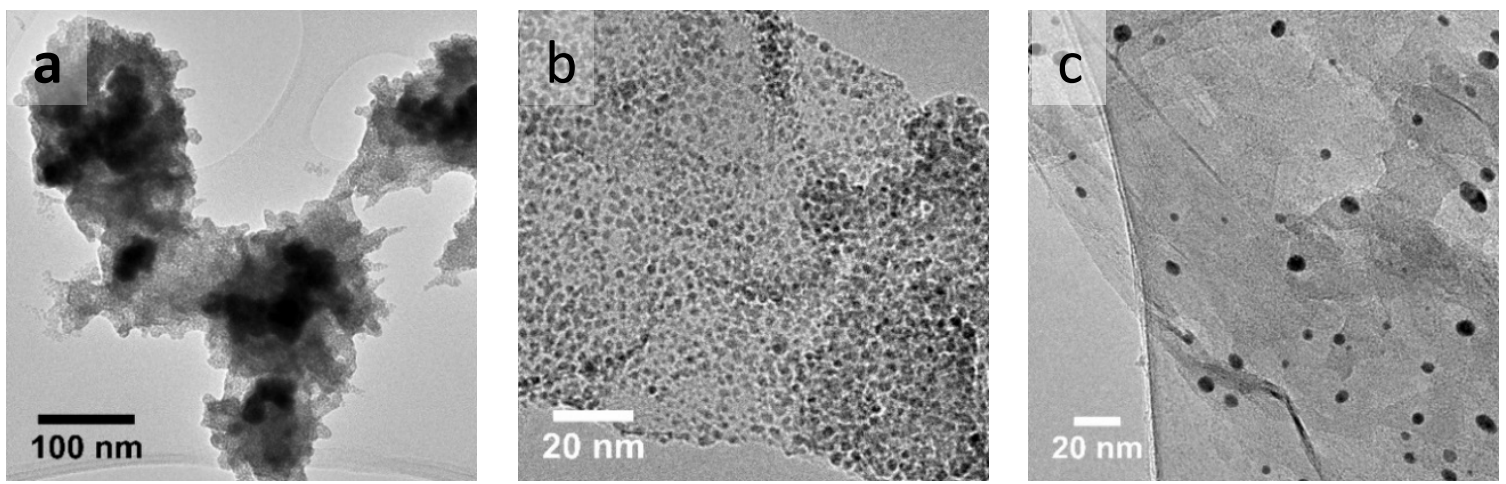

Figure 1. Low magnification TEM images of (a) Pd/Au NPs (b) Pd NPs and (c) Au NPs on graphene. When both metals are present, a core-shell structure is observed, which is absent otherwise. Pd's affinity to graphene is also apparent; this is not the case for Au.
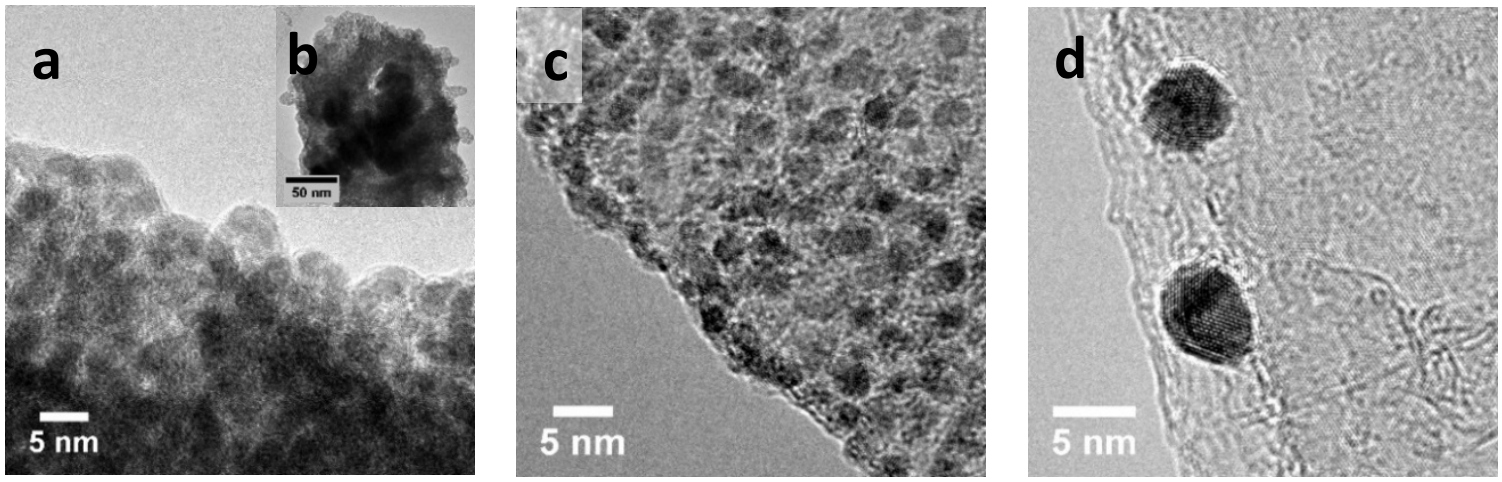

Figure 2. HRTEM images of (a) Pd NPs from an edge of a Pd/Au cluster, as seen in the inset (b). Pd NPs on graphene (c), and Au NPs on graphene (d). The lattice structures confirm crystallinity of the NPs.
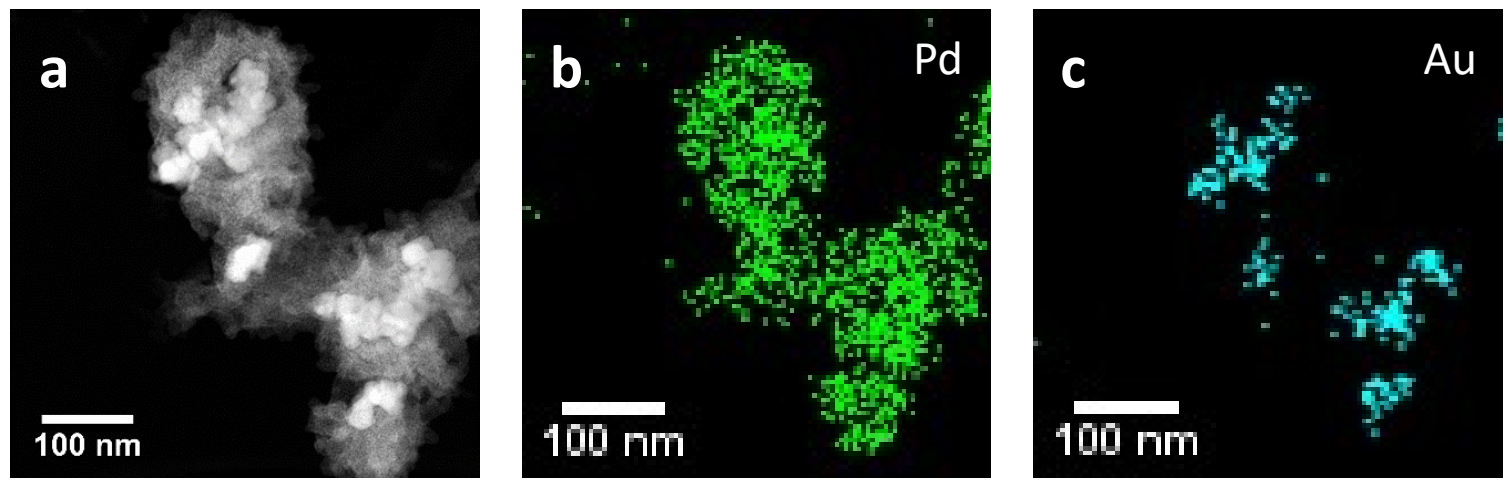

Figure 3. (a) STEM dark-field image of a Pd/Au NP cluster. (b) Pd and (c) Au STEM EDS maps of (a). These images confirm that the cluster is made of $\mathrm{Pd}_{\text {shell }} \mathrm{Au}_{\text {core }}$. 